D.T. Wickramasinghe, L. Ferrario, and G.V. Bicknell, eds.

\title{
Theory and Simulation of Asymmetrically Perturbed Radiatively Cooled Jets
}

\author{
P. E. Hardee \\ Department of Physics $\mathcal{E}$ Astronomy, University of Alabama, \\ Tuscaloosa, AL 35487, U.S.A.
}

J. M. Stone and J. Xu

Department of Astronomy, University of Maryland, College Park, MD 20742, U.S.A.

\begin{abstract}
.
Results of a spatial stability analysis and of numerical simulations of a "slab" jet in which optically thin radiative cooling is dynamically important are presented. Two different cooling curves are used. Unstable Kelvin-Helmholtz modes are significantly different from the adiabatic limit, and the form of the cooling function strongly affects the results. The numerical simulations are in excellent agreement with the linear stability analysis. In the non-linear regime growth of the surface wave at low frequencies results in sinusoidal oscillation which can disrupt the jet, while non-linear body waves produce low amplitude wiggles within the jet that can result in shocks within the jet. In cooling jets, these shocks can produce dense knots and filaments of cooling gas within the jet, and weak shock "spurs" in the ambient gas. Acceleration of ambient gas can be produced by these "spurs", or by rapid entrainment if the jet is disrupted. For parameters typical of protostellar jets perturbations with a period of $<100 \mathrm{yrs}$ should excite body waves which produce internal shocks and small amplitude wiggles. The lack of large amplitude wiggles in most observed systems is consistent with the suggestion that jets arise from the inner regions $(r<1 \mathrm{AU})$ of accretion disks.
\end{abstract}

\section{Introduction}

While many protostellar jets appear remarkably straight, spectacular new images of objects such as HH47 and HH111 (Heathcote et al. 1996; Reipurth et al. 1996; cf, John Bally \& Pat Hartigan, these proceedings) reveal the presence of dramatic kinks and other asymmetric structure. Such observations motivate us to study the development of asymmetries in radiatively cooled jets. Provided jets are not ballistic (i.e., have densities less than 100 times the density of the external material with which the collimated flow interacts) and do not suffer collision with dense clouds, the development of asymmetric structure proceeds by the growth of waves on the Kelvin-Helmholtz unstable jet. In this preliminary study we have compared and contrasted the development of asymmetries on $2 \mathrm{D}$ 
adiabatic and radiatively cooled "slab" jets. A slab jet is spatially resolved along two Cartesian axes and is infinite in extent in the third dimension. By confining our preliminary studies to $2 \mathrm{D}$, we have been able to perform a parameter survey more efficiently.

We have chosen a jet density $n_{j t}=600 \mathrm{~cm}^{-3}$, a jet temperature $T_{j t}=$ $1000 \mathrm{~K}$, a jet radius $R=2.5 \times 10^{15} \mathrm{~cm}$ and considered jet velocities $u=59-$ $236 \mathrm{~km} \mathrm{~s}^{-1}$. The higher velocity is more representative of protostellar jets. With a jet to external medium density ratio $\rho_{j t} / \rho_{e x}=10$ and pressure balance with the external medium, the jet Mach number $M_{e x} \equiv u / a_{e x}=5-20$. In our study we considered the effect of radiative cooling curves for interstellar gas as given by Dalgarno \& McCray (1972, hereafter DM), and for photoionized gas of reduced metallicity as given by MacDonald \& Bailey (1981, hereafter MB). The most important difference between these cooling curves proves to be the temperature dependence where DM cooling has a power law temperature dependence of $\sim T^{0.5}$ on either side of a discontinuity at $10,000 \mathrm{~K}$ and where MB cooling has a power law temperature dependence of $\sim T^{2.5}$.

\section{Theory}

A slab jet is unstable to a symmetric "pinch" mode nearly identical quantitatively to the pinch mode of a 3D cylindrical jet, and an asymmetric "sinusoidal" mode that is qualitatively similar to the helical mode on a $3 \mathrm{D}$ cylindrical jet (Hardee \& Norman 1988). Each mode contains a "surface" (S) wave and multiple "body" (Bn) wave solutions. A complete analysis may be found in Hardee \& Stone (1996). In Figure 1 we show solutions for the sinusoidal mode for DM and for MB type cooling. In general, we find that the inclusion of DM cooling in-
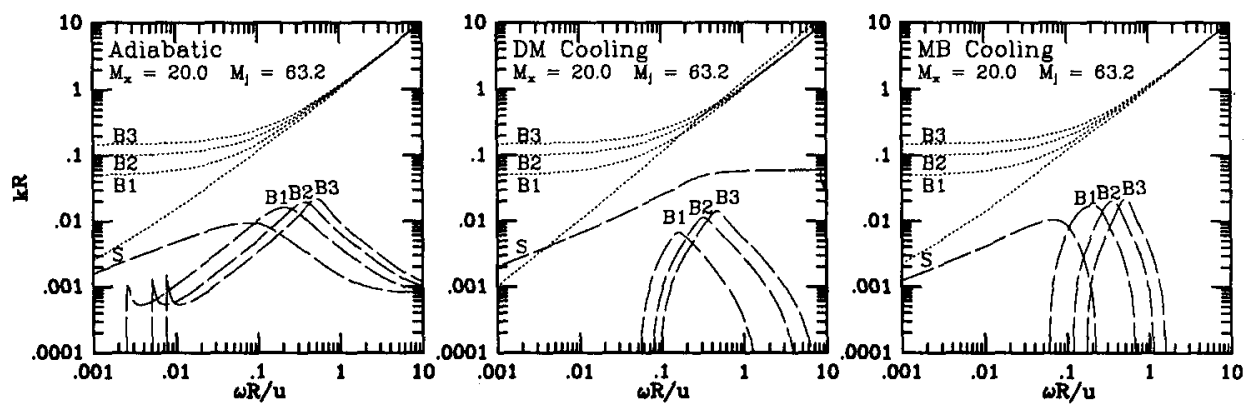

Figure 1. Surface (S) and first three body wave $(\mathrm{Bn})$ solutions for the asymmetric sinusoidal mode. Dashed lines give the imaginary part of the wavenumber. The wavenumber and frequency axes are scaled to different Mach numbers through multiplication by $M_{e x}^{-1}$.

creases the growth rate of the surface wave and decreases the growth rate of the body waves relative to an adiabatic jet. The steep temperature dependence of $\mathrm{MB}$ cooling leads to strong damping effects and, in general, the frequency range over which surface and body waves grow is reduced relative to an adiabatic jet while the maximum growth rates are similar to their values on an adiabatic jet. 
This result is insensitive to Mach number. Computation of fluid displacement surfaces associated with the surface and body waves shows that at frequencies $\omega>2 a_{e x} / R-\omega>0.1 u / R$ (Mach 20 jet) or $\omega>0.4 u / R$ (Mach 5 jet) - only internal oscillation and small amplitude surface oscillation occur.

\section{Simulation}

The simulations are initialized by establishing a slab jet in equilibrium with an external medium across a two-dimensional Cartesian grid. This is equivalent to studying jet flow far behind the jet front. A very small amplitude sinusoidal transverse velocity perturbation is used to excite the asymmetric mode. Dramatic differences between adiabatic, DM cooling, and MB cooling jets are illustrated in Figure 2. Low resolution was used to artificially suppress body

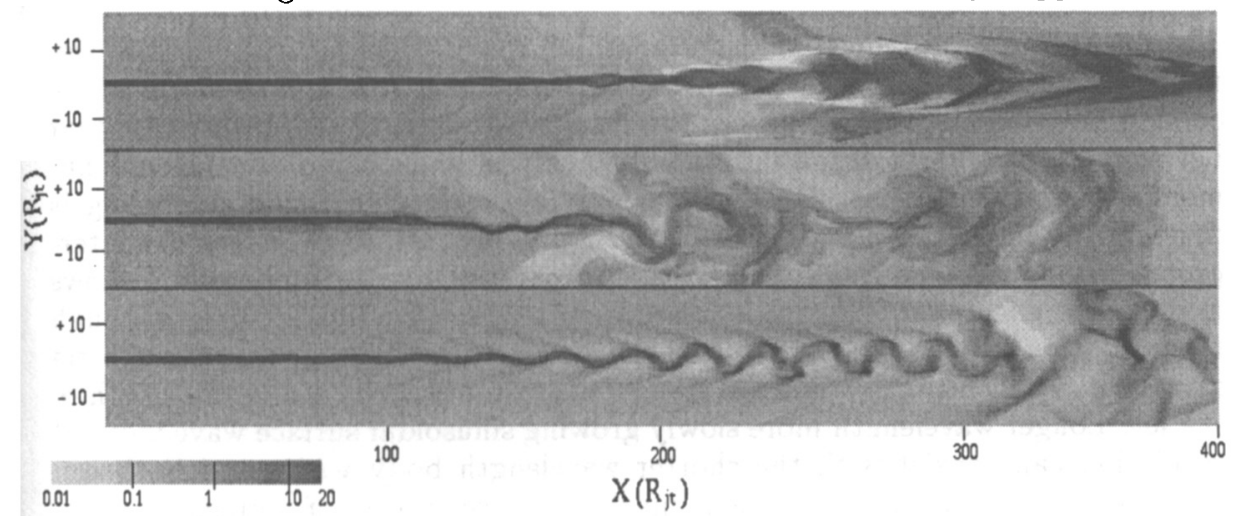

Figure 2. Density images for Mach 5 adiabatic (top), DM cooling (middle), and MB cooling (bottom) jet perturbed with a high frequency of $\omega=5 a_{e x} / R$.

waves. The regular sinusoidal oscillation of the MB cooling jet occurs near the highest unstable frequency whereas adiabatic and DM cooling jets show different behavior appropriate to the different high frequency linear growth rates (for details see Stone, Xu, \& Hardee 1996).

Figure 3 below shows equally dramatic differences between adiabatic and DM cooling jets for a velocity more representative of protostellar jets. In these higher resolution simulations it is possible to see internal asymmetric shocks associated with the body waves. These shocks can produce asymmetric dense knots of cooling gas within the jet. Note the production of shock spurs in the external gas. These shock spurs accelerate the ambient gas. In these simulations we find very little mixing of jet and ambient gas unless the jet is disrupted as in the DM cooling jet in Figure 3. Note that the DM cooling jet forms high density knots associated with the disruption as a result of strong cooling effects. These knots may contain jet and ambient fluid. 


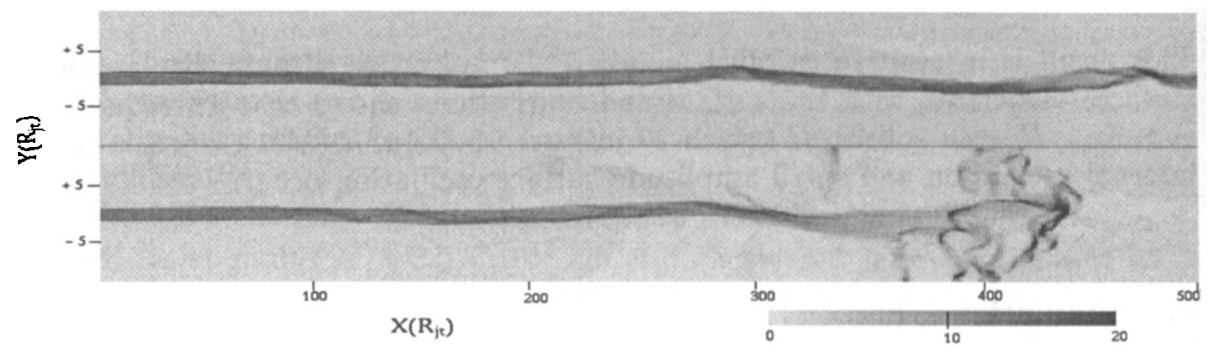

Figure 3. Density images for Mach 20 adiabatic (top) and DM cooling (bottom) jets perturbed with a low frequency of $\omega=0.5 a_{e x} / R$.

\section{Conclusions}

The linear analysis predicts that the unstable Kelvin-Helmholtz modes are significantly different on adiabatic and radiatively cooling jets, and the form of the cooling function strongly influences the results. We have found good agreement between the results of a spatial stability analysis and of two dimensional numerical simulations of a "slab" jet in which optically thin radiative cooling is dynamically important. The linear analysis provides a framework for the interpretation of the numerical simulations. An extensive set of simulations shows that cooling effects can enhance jet disruption relative to adiabatic flows through the formation of dense knots. Internal shocks produced by body waves can form a pattern of dense knots which are staggered asymmetrically along the length of the jet. Longer wavelength more slowly growing sinusoidal surface wave induced oscillation can coexist with the shorter wavelength body waves and both can produce shock spurs in the ambient medium. Acceleration of ambient gas can be produced by these spurs, or by rapid entrainment if the jet is disrupted.

The low amplitude oscillations, the formation of asymmetric knots in the jet and the shock spurs driven into the ambient gas seen in the simulations are reminiscent of features seen in HST observations of protostellar jets such as HH111 (Reipurth et al. 1996). For parameters typical of protostellar jets, the jet cannot respond dynamically to perturbations with a period of $<100 \mathrm{yrs}$. Such perturbations instead excite body waves which produce internal shocks and small amplitude wiggles. The lack of large amplitude wiggles causing disruption of the jet in observed systems indicates that the characteristic timescale of perturbations is short, consistent with the suggestion that jets arise from the inner regions $(r<1 \mathrm{AU})$ of accretion disks.

Acknowledgments. P. Hardee acknowledges support from the NSF through grant AST9318397 and EPSCoR grant OSR-9108761. J. Stone and J. Xu acknowledge support by the NASA through grant NAG-4202.

\section{References}

Dalgarno, A., \& McCray, R.A. 1972, ARA\&A, 10, 375 (DM)

Hardee, P.E., \& Norman, M.L. 1988, ApJ, 334, 70 
Hardee, P.E., \& Stone, J.M. 1996, ApJ, submitted

Heathcote, S., Morse, J., Hartigan, P., Reipurth, B., Schwartz, R.D., Bally, J., \& Stone, J.M. 1996, AJ, in press

MacDonald, J., \& Bailey, M.E. 1981, MNRAS, 197, 995 (MB)

Reipurth, B. et al., 1996, in preparation

Stone, J.M., Xu, J., \& Hardee, P.E. 1996, ApJ, submitted

\section{Discussion}

$J$. $L i$ : Can you conclude that an adiabatic jet is relatively more stable?

P. Hardee: In the linear limit the adiabatic jet can be more or less stable depending on details of the cooling function. However, in the non-linear limit explored by our numerical simulations the jets with radiative cooling are more prone to fragment into dense knots. 